\title{
Beam normal spin asymmetries: Theory
}

\author{
M. Vanderhaeghen ${ }^{\mathrm{a}}$ \\ Department of Physics, College of William and Mary, Williamsburg, VA 23187, USA, and \\ Thomas Jefferson National Accelerator Facility, Newport News, VA 23606, USA \\ Received: 31 August 2006 \\ Published online: 3 July 2007 - (c) Società Italiana di Fisica / Springer-Verlag 2007
}

\begin{abstract}
The beam normal spin asymmetry in elastic electron-nucleon scattering is discussed. This beam normal spin asymmetry depends on the imaginary part of two-photon exchange processes between electron and nucleon, and measures the non-forward structure functions of the nucleon. After briefly reviewing the theoretical formalism, we discuss calculations in the threshold region, in the resonance region, as well as in the diffractive region, corresponding with high energy and forward angles.
\end{abstract}

PACS. 25.30.Bf Elastic electron scattering $-24.70 .+\mathrm{s}$ Polarization phenomena in reactions

\section{Introduction}

Elastic electron-nucleon scattering in the one-photon exchange approximation is a time-honoured tool to access information on the structure of hadrons. Experiments with increasing precision have become possible in recent years, mainly triggered by new techniques to perform polarization experiments at the electron scattering facilities. This has allowed to reach a new frontier in the measurement of hadron structure quantities, such as its electroweak form factors, parity-violating effects, nucleon polarizabilities, transition form factors, or the measurement of spin dependent structure functions, to name a few. For example, experiments using polarized electron beams and measuring the ratio of the recoil nucleon in-plane polarization components have profoundly extended our understanding of the nucleon electromagnetic form factors. For the proton, such polarization experiments which access the ratio $G_{E p} / G_{M p}$ of the proton's electric $\left(G_{E p}\right)$ to magnetic $\left(G_{M p}\right)$ form factors have been performed out to a momentum transfer $Q^{2}$ of $5.6 \mathrm{GeV}^{2}[1-3]$. It came as a surprise that these experiments extracted a ratio of $G_{E p} / G_{M p}$ which is clearly at variance with unpolarized measurements [4-6] using the Rosenbluth separation technique.

The understanding of this puzzle has generated a lot of activity recently, and is a prerequisite to use electron scattering as a precision tool. One possible explanation for the discrepancy between the Rosenbluth and polarization methods is the presence of two-photon exchange effects, beyond those which have already been accounted for in the standard treatment of radiative corrections. A general study of two- (and multi)-photon exchange contributions to the elastic electron-proton scattering observables was

\footnotetext{
a e-mail: marcvdh@jlab.org
}

given in [7]. In that work, it was noted that the interference of the two-photon exchange amplitude with the onephoton exchange amplitude could be comparable in size to the $\left(G_{E}^{p}\right)^{2}$ term in the unpolarized cross-section at large $Q^{2}$. In contrast, the two-photon exchange effects do not impact the polarization-transfer extraction of $G_{E} / G_{M}$ in an equally significant way. Thus a missing and unfactorizable part of the two-photon exchange amplitude at the level of a few percent may well explain the discrepancy between the two methods.

Realistic calculations of elastic electron-nucleon scattering beyond the Born approximation are required in order to demonstrate in a quantitative way that $2 \gamma$ exchange effects are indeed able to resolve this discrepancy.

Recently, several model calculations of the $2 \gamma$ exchange amplitude have been performed. In ref. [8], a calculation of the $2 \gamma$ exchange when the hadronic intermediate state is a nucleon was performed. It found that the $2 \gamma$ exchange correction with intermediate nucleon can partially resolve the discrepancy between the two experimental techniques. It was found [9] however that the effect is partly cancelled when including the next hadronic intermediate state, the $\Delta(1232)$-resonance. The $2 \gamma$ exchange contribution to elastic $e N$ scattering has also been estimated at large momentum transfer $[10,11]$, through the scattering off a parton in a proton by relating the process on the nucleon to the generalized parton distributions. This approach effectively sums all possible excitations of inelastic nucleon intermediate states. It was found that the two-photon corrections to the Rosenbluth process indeed can substantially reconcile the two ways of measuring $G_{E} / G_{M}$.

To push the precision frontier further in electron scattering, one needs a good control of $2 \gamma$ exchange mechanisms and needs to understand how they may or may 
not affect different observables. This justifies a systematic study of such $2 \gamma$ exchange effects, both theoretically and experimentally. The real (dispersive) part of the $2 \gamma$ exchange amplitude can be accessed through the difference between elastic electron and positron scattering off a nucleon. The imaginary (absorptive) part of the $2 \gamma$ exchange amplitude, on the other hand, can be accessed through a single spin asymmetry (SSA) in elastic electron-nucleon scattering, when either the target or beam spin are polarized normal to the scattering plane, as has been discussed some time ago [12]. As time reversal invariance forces this SSA to vanish for one-photon exchange, it is of order $\alpha=e^{2} /(4 \pi) \simeq 1 / 137$. Furthermore, to polarize an ultra-relativistic particle in the direction normal to its momentum involves a suppression factor $m / E$ (with $m$ the mass and $E$ the energy of the particle), which typically is of order $10^{-4}-10^{-3}$ when the electron beam energy is in the $1 \mathrm{GeV}$ range. Therefore, the resulting target normal SSA can be expected to be of order $10^{-2}$, whereas the beam normal SSA is of order $10^{-6}-10^{-5}$. A measurement of such small asymmetries is quite demanding experimentally. However, in the case of a polarized lepton beam, asymmetries of the order ppm are currently accessible in parity violation (PV) elastic $e N$ scattering experiments. The parity-violating asymmetry involves a beam spin polarized along its momentum. However, the SSA for an electron beam spin normal to the scattering plane can also be measured using the same experimental set-ups. First measurements of this beam normal SSA at beam energies up to $1 \mathrm{GeV}$ have yielded values around $-10 \mathrm{ppm}[13-15]$ in the forward angular range and up to an order of magnitude larger in the backward angular range [16]. At higher beam energies, first results for the beam normal SSA in elastic electron-nucleon scattering experiments have also been reported at this meeting $[15,17,18]$.

First estimates of the target normal SSA in elastic electron-nucleon scattering have been performed in $[12$, 19]. In those works, the $2 \gamma$ exchange with nucleon intermediate state (so-called elastic or nucleon pole contribution) has been calculated, and the inelastic contribution has been estimated in a very forward-angle approximation. Estimates within this approximation have also been reported for the beam normal SSA [20]. The general formalism for elastic electron-nucleon scattering with lepton helicity flip, which is needed to describe the beam normal SSA, has been developed in [21]. Furthermore, the beam normal SSA has also been estimated at large momentum transfers $Q^{2}$ in [21] using a parton model, which was found crucial [10] to interpret the results from unpolarized electron-nucleon elastic scattering, as discussed before. In the handbag model of refs. [10,11,21], the corresponding $2 \gamma$ exchange amplitude has been expressed in terms of generalized parton distributions, and the real and imaginary parts of the $2 \gamma$ exchange amplitude are related through a dispersion relation. Hence in the partonic regime, a direct comparison of the imaginary part with experiment can provide a very valuable cross-check on the calculated result for the real part.

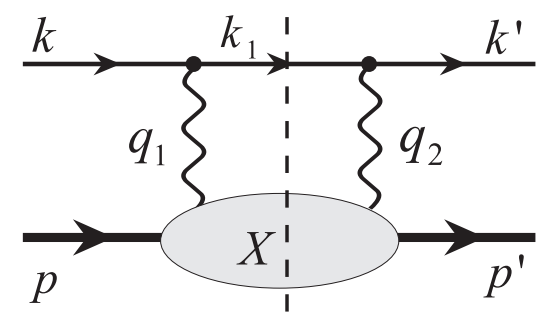

Fig. 1. The two-photon exchange diagram. The filled blob represents the response of the nucleon to the scattering of the virtual photon. In the imaginary part of the two-photon amplitude, the intermediate state indicated by the vertical dashed line is on-shell.

To use the elastic electron-nucleon scattering at low momentum transfer as a high precision tool, such as in present-day PV experiments, one may also want to quantify the $2 \gamma$ exchange amplitude. To this aim, one may envisage a dispersion formalism for the elastic electronnucleon scattering amplitudes, as has been discussed some time ago in the literature [22]. To develop this formalism, the necessary first step is a precise knowledge of the imaginary part of the two-photon exchange amplitude, which enters in both the beam and target normal SSA. Using unitarity, one can relate the imaginary part of the $2 \gamma$ amplitude to the electro-absorption amplitudes on a nucleon, see fig. 1 .

The imaginary part of the $2 \gamma$ exchange has been studied recently in several approaches, both in the threshold region [23], in the resonance region [24], as well as at forward angles in the diffractive region [25-27].

Finally, by understanding the $2 \gamma$ exchange contributions for the case of electromagnetic electron-nucleon scattering, one may extend this calculation to electroweak processes, where the $\gamma Z$ and $W^{+} W^{-}$box diagrams are in several cases the leading unknown contributions entering electroweak precision experiments.

\section{Elastic electron-nucleon scattering beyond the one-photon exchange approximation}

In this section, we briefly review the elastic electronnucleon scattering formalism beyond the one-photon exchange approximation, as has been developed in refs. [7, 21]. For the kinematics of elastic electron-nucleon scattering;

$$
e^{-}(k)+N(p) \rightarrow e^{-}\left(k^{\prime}\right)+N\left(p^{\prime}\right),
$$

we adopt the definitions

$$
P=\frac{p+p^{\prime}}{2}, \quad K=\frac{k+k^{\prime}}{2}, \quad q=k-k^{\prime}=p^{\prime}-p
$$

and choose

$$
Q^{2}=-q^{2}, \quad \nu=K \cdot P
$$

as the independent invariants of the scattering. The invariant $\nu$ is related to the polarization parameter $\varepsilon$ of the 
virtual photon, which can be expressed as (neglecting the electron mass):

$$
\varepsilon=\frac{\nu^{2}-M^{4} \tau(1+\tau)}{\nu^{2}+M^{4} \tau(1+\tau)},
$$

where $M$ is the nucleon mass.

For a theory which respects Lorentz, parity and charge conjugation invariance, the general amplitude for elastic scattering of two spin-( $1 / 2)$ particles can be expressed by 6 independent helicity amplitudes or equivalently by six invariant amplitudes. The total amplitude can be decomposed in general in terms of a lepton spin non-flip and spin flip part:

$$
T=T_{\text {non-flip }}+T_{\text {flip }} .
$$

The non-flip amplitude which conserves the helicity of the electron (in the limit $m_{e}=0$ ) depends upon 3 invariant amplitudes, and has been parametrized in ref. [7] as

$$
\begin{aligned}
T_{\text {non-flip }}= & \frac{e^{2}}{Q^{2}} \bar{u}\left(k^{\prime}\right) \gamma_{\mu} u(k) \\
& \times \bar{u}\left(p^{\prime}\right)\left(\tilde{G}_{M} \gamma^{\mu}-\tilde{F}_{2} \frac{P^{\mu}}{M}+\tilde{F}_{3} \frac{\gamma \cdot K P^{\mu}}{M^{2}}\right) u(p),
\end{aligned}
$$

where $u(k), u\left(k^{\prime}\right)$ and $u(p), u\left(p^{\prime}\right)$ are the lepton and nucleon spinors, respectively. The amplitude which flips the electron helicity (i.e. is of the order of the mass of the electron, $m_{e}$ ), depends on 3 additional invariants which have been introduced in ref. [21] as

$$
\begin{aligned}
& T_{\text {flip }}=\frac{m_{e}}{M} \frac{e^{2}}{Q^{2}}\left[\bar{u}\left(k^{\prime}\right) u(k) \cdot \bar{u}\left(p^{\prime}\right)\left(\tilde{F}_{4}+\tilde{F}_{5} \frac{\gamma \cdot K}{M}\right) u(p)\right. \\
& \left.+\tilde{F}_{6} \bar{u}\left(k^{\prime}\right) \gamma_{5} u(k) \cdot \bar{u}\left(p^{\prime}\right) \gamma_{5} u(p)\right] .
\end{aligned}
$$

In eqs. (6), (7), $\tilde{G}_{M}, \tilde{F}_{2}, \tilde{F}_{3}, \tilde{F}_{4}, \tilde{F}_{5}, \tilde{F}_{6}$ are complex functions of $\nu$ and $Q^{2}$, and the factor $e^{2} / Q^{2}$ has been introduced for convenience. Furthermore in eq. (7), we extracted an explicit factor $m_{e} / M$ out of the amplitudes, which reflects the fact that for a vector interaction (such as in QED), the electron helicity flip amplitude vanishes when $m_{e} \rightarrow 0$. In the Born approximation, one obtains

$$
\begin{aligned}
\tilde{G}_{M}^{\text {Born }}\left(\nu, Q^{2}\right) & =G_{M}\left(Q^{2}\right), \\
\tilde{F}_{2}^{B o r n}\left(\nu, Q^{2}\right) & =F_{2}\left(Q^{2}\right), \\
\tilde{F}_{3,4,5,6}^{B o r n}\left(\nu, Q^{2}\right) & =0,
\end{aligned}
$$

where $G_{M}\left(F_{2}\right)$ are the proton magnetic (Pauli) form factors, respectively.

\section{Single spin asymmetries in elastic electron- nucleon scattering}

An observable which is directly proportional to the two(or multi-) photon exchange is given by the elastic scattering of an unpolarized electron on a proton target polarized normal to the scattering plane (or the recoil polarization normal to the scattering plane, which is exactly the same assuming time-reversal invariance). For a target polarized perpendicular to the scattering plane, the corresponding single spin asymmetry, which we refer to as the target normal spin asymmetry $\left(A_{n}\right)$, is defined by

$$
A_{n}=\frac{\sigma_{\uparrow}-\sigma_{\downarrow}}{\sigma_{\uparrow}+\sigma_{\downarrow}},
$$

where $\sigma_{\uparrow}\left(\sigma_{\downarrow}\right)$ denotes the cross-section for an unpolarized beam and for a nucleon spin parallel (anti-parallel) to the normal polarization vector, defined as

$$
S_{n}^{\mu}=\left(0, \boldsymbol{S}_{n}\right), \quad \boldsymbol{S}_{n} \equiv\left(\boldsymbol{k} \times \boldsymbol{k}^{\prime}\right) /\left|\boldsymbol{k} \times \boldsymbol{k}^{\prime}\right| .
$$

As has been shown by de Rujula et al. [12], the target (or recoil) normal spin asymmetry is related to the absorptive part of the elastic $e N$ scattering amplitude as

$$
A_{n}=\frac{2 \operatorname{Im}\left(\sum_{\text {spins }} T_{1 \gamma}^{*} \cdot \operatorname{Abs} T_{2 \gamma}\right)}{\sum_{\text {spins }}\left|T_{1 \gamma}\right|^{2}},
$$

where $T_{1 \gamma}$ denotes the one-photon exchange amplitude. Since the one-photon exchange amplitude is purely real, the leading contribution to $A_{n}$ is of order $O\left(e^{2}\right)$, and is due to an interference between one- and two-photon exchange amplitudes.

For a beam polarized perpendicular to the scattering plane, one can also define a single spin asymmetry, analogously as in eq. (9) as noted in ref. [20], where now $\sigma_{\uparrow}\left(\sigma_{\downarrow}\right)$ denotes the cross section for an unpolarized target and for an electron beam spin parallel (anti-parallel) to the normal polarization vector, given by eq. (10). We refer to this asymmetry as the beam normal spin asymmetry $\left(B_{n}\right)$. It explicitly vanishes when $m_{e}=0$ as it involves an electron helicity flip. Using the general electron-nucleon scattering amplitude of eqs. (6), (7), $B_{n}$ is given by [21]

$$
\begin{aligned}
B_{n}= & \frac{2 m_{e}}{Q} \sqrt{2 \varepsilon(1-\varepsilon)} \sqrt{1+\frac{1}{\tau}}\left(G_{M}^{2}+\frac{\varepsilon}{\tau} G_{E}^{2}\right)^{-1} \\
& \times\left\{-\tau G_{M} \mathcal{I}\left(\tilde{F}_{3}+\frac{1}{1+\tau} \frac{\nu}{M^{2}} \tilde{F}_{5}\right)\right. \\
& \left.\quad-G_{E} \mathcal{I}\left(\tilde{F}_{4}+\frac{1}{1+\tau} \frac{\nu}{M^{2}} \tilde{F}_{5}\right)\right\}+\mathcal{O}\left(e^{4}\right),
\end{aligned}
$$

with $\tau \equiv Q^{2} /\left(4 M^{2}\right)$. As for $A_{n}$, also $B_{n}$ vanishes in the Born approximation, and is therefore of order $e^{2}$.

\section{Imaginary (absorptive) part of the two-photon exchange amplitude}

In this section we discuss the relation between the imaginary part of the two-photon exchange amplitude and the absorptive part of the doubly virtual Compton scattering tensor on the nucleon, as shown in fig. 1. In the following we consider the helicity amplitudes for the elastic electronnucleon scattering, defined in the $e^{-} N$ c.m. frame, which 
are denoted by $T\left(h^{\prime}, \lambda_{N}^{\prime} ; h, \lambda_{N}\right)$. Here $h\left(h^{\prime}\right)$ denote the helicities of the initial (final) electrons and $\lambda_{N}\left(\lambda_{N}^{\prime}\right)$ denote the helicities of the initial (final) nucleons.

The discontinuity of the two-photon exchange amplitude, shown in fig. 1, can then be expressed as

$$
\begin{aligned}
\operatorname{Abs}_{2 \gamma}= & e^{4} \int \frac{\mathrm{d}^{3} \boldsymbol{k}_{1}}{(2 \pi)^{3} 2 E_{k_{1}}} \bar{u}\left(k^{\prime}, h^{\prime}\right) \gamma_{\mu}\left(\gamma \cdot k_{1}+m_{e}\right) \gamma_{\nu} u(k, h) \\
& \times \frac{1}{Q_{1}^{2} Q_{2}^{2}} \cdot W^{\mu \nu}\left(p^{\prime}, \lambda_{N}^{\prime} ; p, \lambda_{N}\right),
\end{aligned}
$$

where the momenta are defined as indicated in fig. 1, with $q_{1} \equiv k-k_{1}, q_{2} \equiv k^{\prime}-k_{1}$, and $q_{1}-q_{2}=q$. Denoting the c.m. angle between initial and final electrons as $\theta_{c . m}$., the momentum transfer $Q^{2} \equiv-q^{2}$ in the elastic-scattering process can be expressed as

$$
Q^{2}=\frac{\left(s-M^{2}\right)^{2}}{2 s}\left(1-\cos \theta_{c . m .}\right)+\mathcal{O}\left(m_{e}^{2}\right),
$$

with $s=(k+p)^{2}$. Furthermore, $Q_{1}^{2} \equiv-q_{1}^{2}=-(k-$ $\left.k_{1}\right)^{2}$ and $Q_{2}^{2} \equiv-q_{2}^{2}=-\left(k^{\prime}-k_{1}\right)^{2}$ correspond with the virtualities of the two exchanged spacelike photons.

In eq. (13), the hadronic tensor $W^{\mu \nu}\left(p^{\prime}, \lambda_{N}^{\prime} ; p, \lambda_{N}\right)$ corresponds with the absorptive part of the doubly virtual Compton scattering tensor with two space-like photons:

$$
\begin{aligned}
& W^{\mu \nu}\left(p^{\prime}, \lambda_{N}^{\prime} ; p, \lambda_{N}\right)=\sum_{X}(2 \pi)^{4} \delta^{4}\left(p+q_{1}-p_{X}\right) \\
& \quad \times\left\langle p^{\prime}, \lambda_{N}^{\prime}\left|J^{\dagger \mu}(0)\right| X\right\rangle\left\langle X\left|J^{\nu}(0)\right| p, \lambda_{N}\right\rangle,
\end{aligned}
$$

where the sum goes over all possible on-shell intermediate hadronic states $X$. Note that in the limit $p^{\prime}=$ $p$, eq. (15) reduces to the forward tensor for inclusive electron-nucleon scattering and can be parametrized by the usual 4 nucleon forward structure functions. In the non-forward case however, the absorptive part of the doubly virtual Compton scattering tensor of eq. (15) which enters in the evaluation of target and beam normal spin asymmetries, depends upon 18 invariant amplitudes [28]. Though this may seem as a forbiddingly large number of new functions, we may use the unitarity relation to express the full non-forward tensor in terms of electroproduction amplitudes $\gamma^{*} N \rightarrow X$. The number of intermediate states $X$ which one considers in the calculation will then put a limit on how high in energy one can reliably calculate the hadronic tensor eq. (15). In the following section, the tensor $W^{\mu \nu}$ will be discussed for the elastic contribution $(X=N)$, in the resonance region as a sum over all $\pi N$ intermediate states (i.e. $X=\pi N)$, using a phenomenological state-of-the-art calculation for the $\gamma^{*} N \rightarrow \pi N$ amplitudes, in the diffractive region (corresponding with highenergy, forward scattering) where it can be related to the total photo-absorption cross-section on a proton, as well as in the hard scattering region where it can be related to nucleon generalized parton distributions.

In fig. 2, the full kinematical accessible region for the virtualities $Q_{1}^{2}, Q_{2}^{2}$ in the phase space integral of eq. (13) is shown. The full phase space integral is three-dimensional, the third variable represents the invariant mass $W$ of the

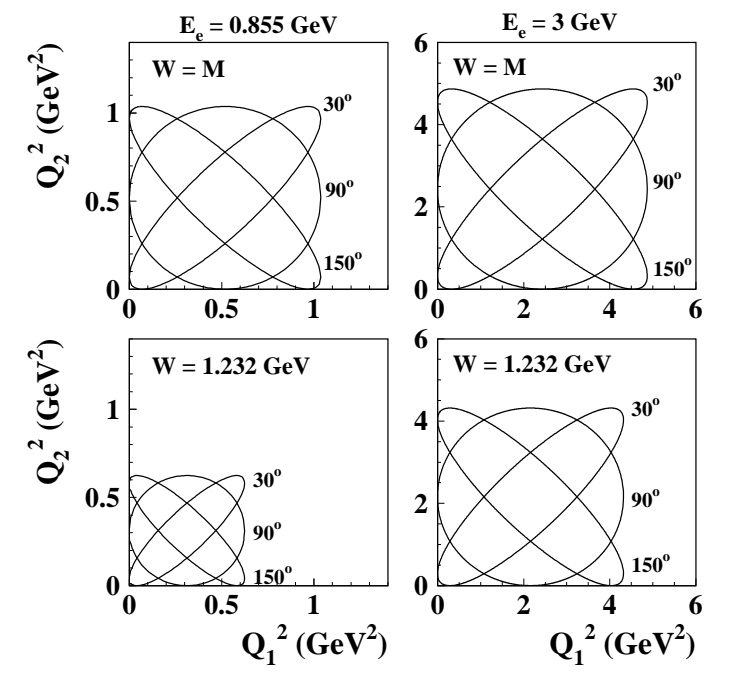

Fig. 2. Kinematical accessible region for the virtualities $Q_{1}^{2}, Q_{2}^{2}$ in the phase space integral of eq. (13), for MAMI (left panels) and JLab (right panels) kinematics for different c.m. angles $\theta_{c . m}$. as indicated. The accessible regions correspond with the inside of the ellipses and are shown both for the elastic (upper panels) and for inelastic (lower panels) intermediate hadronic states. The intersection with the axes correspond with quasiVCS, whereas the situation at $W=\sqrt{s}-m_{e}$ where all ellipses shrink to the point $Q_{1}^{2}=Q_{2}^{2} \simeq 0$ corresponds with quasi-RCS.

intermediate state $X$, defined as $W^{2} \equiv p_{X}^{2}$. There are special regions in this phase space integral (corresponding with near singularities) which may give important contributions (logarithmic enhancements) under some kinematical conditions.

When the intermediate and initial electrons are collinear, then also the photon with momentum $\boldsymbol{q}_{1}=$ $\boldsymbol{k}-\boldsymbol{k}_{1}$ is collinear with this direction. For the elastic case $(W=M)$ this precisely corresponds with the situation where the first photon is soft (i.e. $q_{1} \rightarrow 0$ ) and where the second photon carries the full momentum transfer $Q_{2}^{2} \simeq Q^{2}$. For the inelastic case $(W>M)$ the first photon is hard but becomes quasi-real (i.e. $Q_{1}^{2} \sim m_{e}^{2}$ ). In this case, the virtuality of the second photon is smaller than $Q^{2}$. An analogous situation occurs when the intermediate electron is collinear with the final electron. These kinematical situations with one quasi-real photon and one virtual photon correspond with quasi-virtual Compton scattering (quasi-VCS). Besides the quasi-VCS singularities, the two-photon exchange amplitude also has a near singularity when the intermediate electron momentum is soft (i.e. $\left.\left|\boldsymbol{k}_{1}\right| \rightarrow 0\right)$. In this case the first photon takes on the full momentum of the initial electron, i.e. $\boldsymbol{q}_{1} \rightarrow \boldsymbol{k}$, whereas the second photon takes on the full momentum of the final electron, i.e. $\boldsymbol{q}_{2} \rightarrow \boldsymbol{k}^{\prime}$. This situation occurs when the invariant mass of the hadronic state takes on its maximal value $W_{\max }=\sqrt{s}-m_{e}$. In this case, both photon virtualities become very small and vanish if the electron mass is taken to zero. This kinematical situation with two quasi-real photons corresponds with quasi-real Compton scattering (quasi-RCS). 


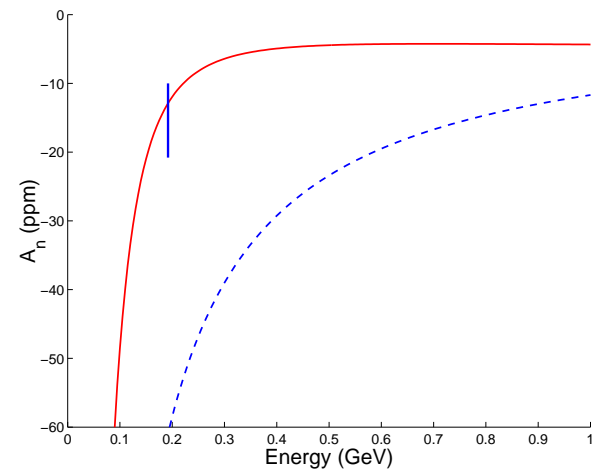

Fig. 3. Beam normal spin asymmetry for $e^{-\uparrow} p \rightarrow e^{-} p$ at fixed c.m. scattering angle $\theta_{c m}=146.1$ degrees. Calculations and figure from ref. [23]. Dashed (solid) curves are the leading order (full) results. The data point is from the SAMPLE Collaboration $[13]$.

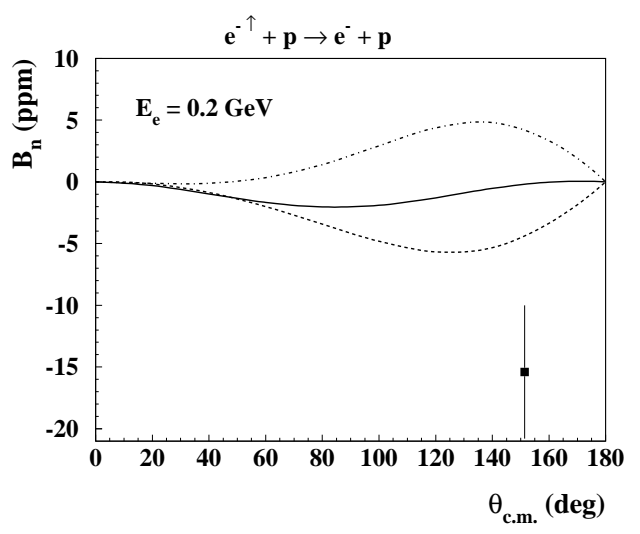

Fig. 4. Beam normal spin asymmetry for $e^{-\uparrow} p \rightarrow e^{-} p$ at a beam energy $E_{e}=0.2 \mathrm{GeV}$ as function of the c.m. scattering angle, for different hadronic intermediate states $(X)$ in the blob of fig. 1: $N$ (dashed curve), $\pi N$ (dashed-dotted curve), sum of the $N$ and $\pi N$ (solid curve). Calculations and figure are from ref. [24]. The data point is from the SAMPLE Collaboration [13].

\section{Results and discussion}

\subsection{Threshold region}

In ref. [23], the beam normal spin asymmetry was studied at low energies in an effective theory of electrons, protons and photons. This calculation, in which pions are integrated out, effectively corresponds with the nucleon intermediate-state contribution only, expanded to second order in $E_{e} / M_{N}$. To this order, the calculation includes the recoil corrections to the scattering from a point charge, the nucleon charge radius, and the nucleon isovector magnetic moment. One sees from fig. 3 that the theory expanded up to second order in $E_{e} / M_{N}$ (indicated by the full results) are able to give a good account of the SAMPLE data point at the low energy $E_{e}=0.2 \mathrm{GeV}$.

When doing the full calculation for the $N$ intermediate state, which is model independent (as it only involves onshell $\gamma^{*} N N$ matrix elements), the result is further reduced

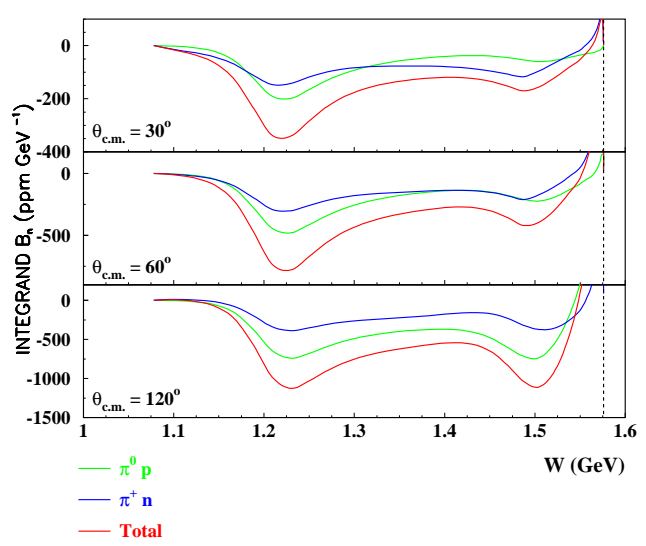

Fig. 5. Integrand in $W$ of $B_{n}$ for $e^{-\uparrow} p \rightarrow e^{-} p$ at a beam energy of $E_{e}=0.855 \mathrm{GeV}$ and at different c.m. scattering angles as indicated on the figure. The vertical dashed line indicates the upper limit of the $W$ integration, i.e. $W_{\max }=\sqrt{s}-m_{e}$. Calculations and figure from ref. [24].

as seen in fig. 4. Inclusion of threshold pion electroproduction contributions, arising from the $\pi N$ intermediate states, partly cancels the elastic contributions. Because in this low-energy region, the matrix elements are rather well known, it is not clear how to get a better agreement with the rather large asymmetry measured by SAMPLE [13].

\subsection{Resonance region}

When measuring the imaginary part of the elastic $e N$ amplitude through a normal SSA at sufficiently low energies, below or around two-pion production threshold, one is in a regime where these electroproduction amplitudes are relatively well known using pion electroproduction experiments as input. As both photons in the $2 \gamma$ exchange process are virtual and integrated over, an observable such as the beam or target normal SSA is sensitive to the electroproduction amplitudes on the nucleon for a range of photon virtualities. This may provide information on resonance transition form factors complementary to the information obtained from current pion electroproduction experiments.

In ref. [24], the imaginary part of the two-photon exchange amplitude was calculated by relating it through unitarity to the contribution of $X=N$ and $X=\pi N$ intermediate state contributions. For the $\pi N$ intermediate state contribution, the corresponding pion electroproduction amplitudes were taken from the phenomenological MAID analysis [29], which contains both resonant and non-resonant pion production mechanisms. As an example, the integrand in $W$ of the phase space integral of eq. (13) is shown in fig. 5 at $E_{e}=0.855 \mathrm{GeV}$. The resonance structure is clearly reflected in the integrands for both $\pi^{+} n$ and $\pi^{0} p$ channels. At forward angles, the quasireal Compton scattering at the endpoint $W=W_{\max }$ only yields a very small contribution, which grows larger when going to backward angles. This quasi-RCS contribution is of opposite sign as the remainder of the integrand, and 

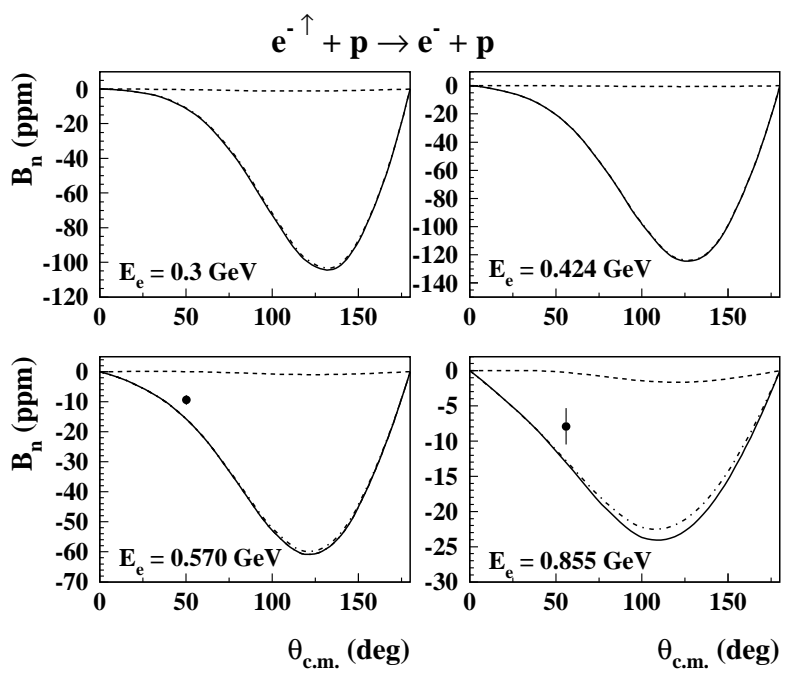

Fig. 6. Beam normal spin asymmetry $B_{n}$ for $e^{-\uparrow} p \rightarrow e^{-} p$ as function of the c.m. scattering angle at different beam energies, as indicated on the figure. The calculations are for different hadronic intermediate states $(X)$ in the blob of fig. 1: $N$ (dashed curve), $\pi N$ (dashed-dotted curves), sum of the $N$ and $\pi N$ (solid curves). The data points are from the A4 Collaboration (MAMI) [14]. Calculations and figure from ref. [24].

therefore determines the position of the maximum (absolute) value of $B_{n}$ when going to backward angles.

In fig. 6 , the results for $B_{n}$ are shown at different beam energies below $E_{e}=1 \mathrm{GeV}$. It is clearly seen that at energies $E_{e}=0.3 \mathrm{GeV}$ and higher the nucleon intermediate state (elastic part) yields only a very small relative contribution. Therefore $B_{n}$ is a direct measure of the inelastic part which gives rise to sizeable large asymmetries, of the order of several tens of ppm in the backward angular range, mainly driven by the quasi-RCS near singularity. First results from the A4 Collaboration for $B_{n}$ at backward angles (for $E_{e}$ around $0.3 \mathrm{GeV}$ ) shown at this meeting [16] indeed show such a large beam normal spin asymmetry of order $-100 \mathrm{ppm}$ for $\theta_{c m}$ around 150 degrees. At forward angles, the size of the predicted asymmetries is compatible with the first high-precision measurements performed at MAMI, though the model slightly overpredicts (in absolute value) $B_{n}$ at $E_{e}=0.57 \mathrm{GeV}$ and $0.855 \mathrm{GeV}$.

\subsection{High-energy, forward-scattering (diffractive) region}

At very high energies and forward-scattering angles (socalled diffractive limit), it was shown in refs. $[25,26]$ that the beam normal spin asymmetry is dominated by the quasi-real Compton singularity. In this (extreme forward limit) case, the hadronic tensor can be expressed in terms of the total photo-absorption cross-section, allowing to express $B_{n}$ through the simple analytic expression:

$$
B_{n}=-\frac{m_{e} \sqrt{Q^{2}} \sigma_{t o t}^{\gamma p}}{8 \pi^{2}} \frac{G_{E}}{\tau G_{M}^{2}+\varepsilon G_{E}^{2}}\left[\log \frac{Q^{2}}{m_{e}^{2}}-2\right] .
$$
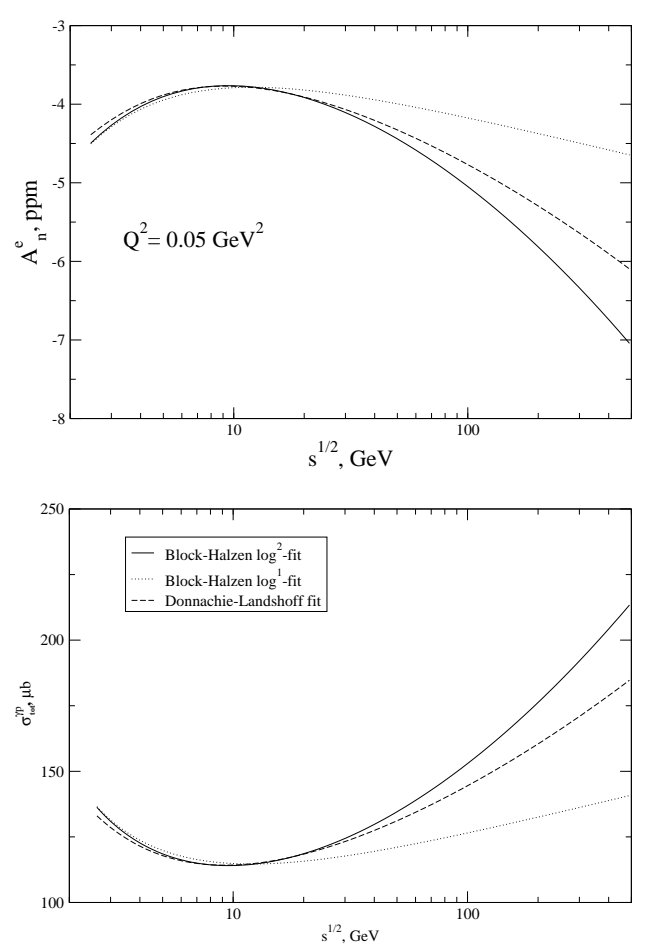

Fig. 7. Beam normal spin asymmetry (top panel) at high energies and very forward angles $\left(Q^{2}=0.05 \mathrm{GeV}^{2}\right)$ using eq. (16) for different parameterizations of the total photo-absorption cross section on the proton (lower panel). Calculations and figure from ref. [25].

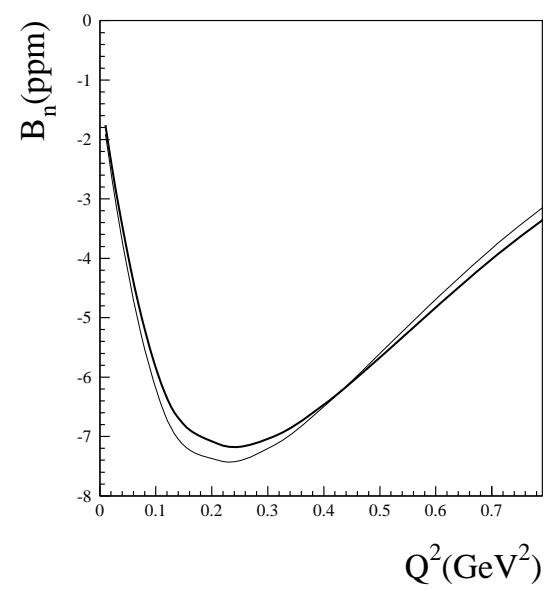

Fig. 8. Beam normal spin asymmetry for elastic $e^{-} p$ scattering for $E_{e}=3 \mathrm{GeV}$. The thick curve is the leading- $Q^{2}$ contribution, whereas the thin line shows the full calculation with subleading terms in $Q^{2}$. Calculations and figure from [27].

One notices that the quasi-real Compton singularity gives rise to a (single) logarithmic enhancement factor which is at the origin of the relatively large value of $B_{n}$.

In fig. 7, the estimate from ref. [25] based on eq. (16) is shown for different parameterizations of the total photoabsorption cross-section. The beam normal spin asymmetry has been measured at SLAC (E-158) at an energy 
Table 1. Forward-angle experimental measurements of the beam normal spin asymmetry.

\begin{tabular}{l|l|l|l}
\hline EXP. & $E_{e}(\mathrm{GeV})$ & $Q^{2}\left(\mathrm{GeV}^{2}\right)$ & $B_{n}(\mathrm{ppm})$ \\
\hline SAMPLE [13] & 0.192 & 0.10 & $-16.4 \pm 5.9$ \\
A4 [14] & 0.570 & 0.11 & $-8.59 \pm 0.89$ \\
A4 [14] & 0.855 & 0.23 & $-8.52 \pm 2.31$ \\
HAPPEX [15] & 3.0 & 0.11 & $-6.7 \pm 1.5$ \\
E-158 [17] & 46 & 0.06 & $-3.5 \rightarrow-2.5$ \\
\hline
\end{tabular}

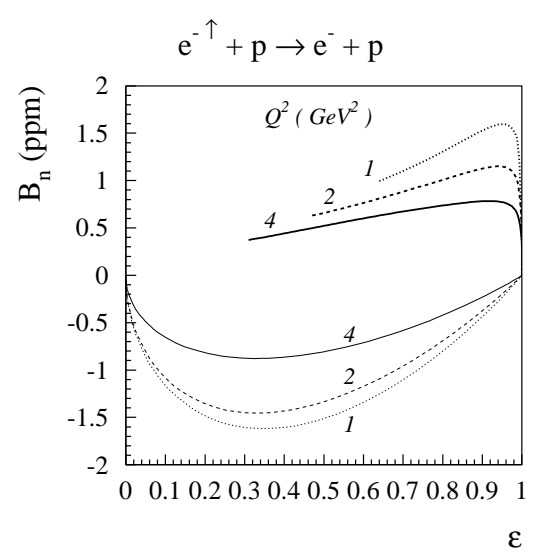

Fig. 9. Beam normal spin asymmetry for elastic $e^{-} p$ scattering as function of $\varepsilon$ at different values of $Q^{2}$ as indicated on the figure. The upper thick curves $\left(B_{n}>0\right)$ are the GPD calculations for the kinematical range where $s,-u>M^{2}$. For comparison, the nucleon pole contribution is also displayed: lower thin curves $\left(B_{n}<0\right)$. Calculations and figure from [21].

$E_{e}=46 \mathrm{GeV}(\sqrt{s} \simeq 9 \mathrm{GeV})$ and very forward angle $\left(Q^{2} \simeq 0.05 \mathrm{GeV}^{2}\right)$. First results, reported at this meeting, indicate a value $B_{n} \simeq-3.5 \rightarrow-2.5 \mathrm{ppm}$, confirming the estimate shown in fig. 7 .

At intermediate energies, around $E_{e} \simeq 3 \mathrm{GeV}$, and forward angles, $B_{n}$ has also been measured by the HAPPEX and G0 Collaborations. The simple "diffractive" formula of eq. (16) does not rigorously apply any more and one has to calculate corrections due to the deviation from forward scattering. Such calculation has recently been performed in [27] and the result is shown in fig. 8. The resulting asymmetries are in basic agreement with first results reported by HAPPEX [15] and G0 [18].

In table 1, a summary of forward-angle measurements of beam normal spin asymmetries is shown.

\subsection{Hard-scattering region}

In the hard-scattering region, the normal beam spin asymmetry was estimated in [21] through the scattering off a parton, which is embedded in the nucleon through a generalized parton distribution. Using phenomenological parametrizations for the GPDs, $B_{n}$ was found to yield values around $+1 \mathrm{ppm}$ to $+1.5 \mathrm{ppm}$ in the few $\mathrm{GeV}$ beam energy range, see fig. 9. In particular, the forward angular range for $e^{-\uparrow} p \rightarrow e^{-} p$ scattering was found to be a favorable region to get information on the inelastic part of $B_{n}$. Because in the handbag calculation, real and imaginary parts are linked, a direct measurement of $B_{n}$ may yield a valuable cross-check for the real part, which was found crucial in understanding the unpolarized cross section data for $e^{-} p \rightarrow e^{-} p$ at large momentum transfer.

\section{References}

1. M.K. Jones et al., Phys. Rev. Lett. 84, 1398 (2000).

2. O. Gayou et al., Phys. Rev. Lett. 88, 092301 (2002).

3. V. Punjabi et al., Phys. Rev. C 71, 055202 (2005); 069902 (2005)(E).

4. L. Andivahis et al., Phys. Rev. D 50, 5491 (1994).

5. M.E. Christy et al., Phys. Rev. C 70, 015206 (2004).

6. I.A. Qattan et al., Phys. Rev. Lett. 94, 142301 (2005).

7. P.A.M. Guichon, M. Vanderhaeghen, Phys. Rev. Lett. 91, 142303 (2003).

8. P.G. Blunden, W. Melnitchouk, J.A. Tjon, Phys. Rev. Lett. 91, 142304 (2003); Phys. Rev. C 72, 034612 (2005).

9. S. Kondratyuk, P.G. Blunden, W. Melnitchouk, J.A. Tjon, Phys. Rev. Lett. 95, 172503 (2005).

10. Y.C. Chen, A. Afanasev, S.J. Brodsky, C.E. Carlson, M. Vanderhaeghen, Phys. Rev. Lett. 93, 122301 (2004).

11. A.V. Afanasev, S.J. Brodsky, C.E. Carlson, Y.C. Chen, M. Vanderhaeghen, Phys. Rev. D 72, 013008 (2005).

12. A. De Rujula, J.M. Kaplan, E. de Rafael, Nucl. Phys. B 35, 365 (1971).

13. SAMPLE Collaboration (S.P. Wells et al.), Phys. Rev. C 63, 064001 (2001).

14. MAMI/A4 Collaboration (F. Maas et al.), Phys. Rev. Lett. 94, 082001 (2005).

15. On behalf of the HAPPEX Collaboration (L. Kaufmann), this conference.

16. On behalf of the A4 Collaboration (L. Capozza), this conference.

17. On behalf of the E-158 Collaboration (K. Kumar), this conference.

18. JLab G0 experiment (E-00-006, E-01-116), spokesperson D. Beck.

19. A. De Rujula, J.M. Kaplan, E. de Rafael, Nucl. Phys. B 53, 545 (1973).

20. A. Afanasev, I. Akusevich, N.P. Merenkov, hep$\mathrm{ph} / 0208260$.

21. M. Gorchtein, P.A.M. Guichon, M. Vanderhaeghen, Nucl. Phys. A 741, 234 (2004).

22. J.A. Peñarrocha, J. Bernabéu, Ann. Phys. 135, 321 (1981); J. Bordes, J.A. Peñarrocha, J. Bernabéu, Phys. Rev. D 35, 3310 (1987).

23. L. Diaconescu, M.J. Ramsey-Musolf, Phys. Rev. C 70, 054003 (2004).

24. B. Pasquini, M. Vanderhaeghen, Phys. Rev. C 70, 045206 (2004).

25. A.V. Afanasev, N.P. Merenkov, Phys. Lett. B 599, 48 (2004) (arXiv:hep-ph/0407167).

26. M. Gorchtein, Phys. Rev. C 73, 035213 ; 055201 (2006).

27. M. Gorchtein, arXiv:hep-ph/0610378.

28. R. Tarrach, Nuovo Cimento A 28, 409 (1975).

29. D. Drechsel, O. Hanstein, S. Kamalov, L. Tiator, Nucl. Phys. A 645, 145 (1999). 